\title{
Barber Pole Sign in CT Angiography, Adult Presentation of Midgut Malrotation: A Case Report
}

\author{
Juan Arsenio Garcelan-Trigo, ${ }^{1, *}$ Manuel Tello-Moreno, ${ }^{1}$ Manuel Jesus Rabaza-Espigares, ${ }^{1}$ and \\ Ildefonso Talavera-Martinez ${ }^{1}$ \\ ${ }^{1}$ Diagnostic Imaging Unit, Hospital San Agustin, Linares (Jaen), Spain \\ *Corresponding author: Juan Arsenio Garcelan Trigo, Diagnostic Imaging Unit, Hospital San Agustin, Linares (Jaen), Spain. Tel: +34-618177483, E-mail: juanarsenio@gmail.com
}

Received: January 26, 2014; Revised: March 21, 2014; Accepted: April 26, 2014

\begin{abstract}
Adult midgut volvulus is a challenging diagnosis because of its low incidence and nonspecific symptoms. Diagnostic delay and longterm complaints are frequent in this clinical scenario. We reported a patient referred to our diagnostic imaging unit with intermittent abdominal pain, bloating and episodic vomiting for several years. He underwent barium gastrointestinal transit and abdominal ultrasound, which revealed severe gastric dilatation, food retention and slow transit until a depressed duodenojejunal flexure, with malrotation of the midgut and jejunal loops being located in the right upper quadrant. Computed tomography angiography was performed, showing rotation of the small intestine around the mesentery root, suggestive of midgut malrotation. In addition, an abnormal twisted disposition of superior mesenteric artery with corkscrew appearance was seen, shaping the pole-barber sign which was evident in volume rendering three-dimensional reconstructions. The patient underwent scheduled surgical treatment without any complication and had good outcome after hospital discharge and follow-up. Computed tomography plays an important role in evaluation of adult midgut volvulus. In addition, angiographic reconstructions can help us to assess the anatomic disposition of mesenteric vascular supply. Both of these assessments are useful in preoperative management.
\end{abstract}

Keywords: Intestines; Intestinal Volvulus; Multidetector Computed Tomography; Adult

\section{Introduction}

Midgut malrotation is an infrequent congenital anomaly, with an estimated incidence of $0.2 \%$ in pediatric population. When symptomatic, it is usually diagnosed within the first months after birth and more than 90\% of symptomatic patients present during the first year of life. Therefore, adult midgut malrotation is a very rare condition, affecting less than $0.16 \%$ of adult population (1). Most of adult patients is detected incidentally during a diagnostic imaging or a surgery. Nevertheless, acute or chronic complications may occur in adults, such as midgut volvulus. Midgut volvulus as the most frequent complication is not an easy diagnosis because of its low incidence and unspecific presentation symptoms, such as abdominal pain, vomiting and food intolerance (2). Diagnostic imaging techniques play an important role in the diagnosis, especially computed tomography (CT) (which reveals the abnormal position of midgut) as well as barium studies of gastrointestinal tract. These techniques can assess the anatomic disposition and mesenteric vascular supply. Barber pole sign was first described by Buranasiri et al. (3) as an angiographic sign of midgut volvulus. We described the diagnosis of a midgut volvulus presenting in adult, with the typical barber pole sign in CT angiography that is better shown in three-dimensional volume rendering reconstructions.

\section{Case Presentation}

A 41-year-old man, with no significant medical history, was referred from a gastroenterologist consult for longterm (more than 10 years) intermittent abdominal pain, bloating and vomiting, mostly 2 - 3 hours after eating. He had no significant analytic abnormalities. The ultrasound imaging revealed severe gastric dilatation, with food and gas retention that prevented proper assessment of retroperitoneal structures (Figure 1). Barium gastrointestinal transit confirmed gastric dilatation extended to the proximal duodenum without bulbar deformity (Figure 2), together with a depressed duodenojejunal flexure and deviation of the midgut usual trajectory. The fourth part of duodenum was downward and the jejunal loops were located in the right upper quadrant. A linear mark corresponding to the Ladd's band was seen (Figure 3) at the duodenojejunal transition and the jejunal loops were not dilated from this point. With suspicious to midgut malrotation, worsening of symptoms led to abdominal CT study request.

Copyright (C) 2015, Tehran University of Medical Sciences and Iranian Society of Radiology. This is an open-access article distributed under the terms of the Creative Commons Attribution-NonCommercial 4.0 International License (http://creativecommons.org/licenses/by-nc/4.0/) which permits copy and redistribute the material just in noncommercial usages, provided the original work is properly cited. 


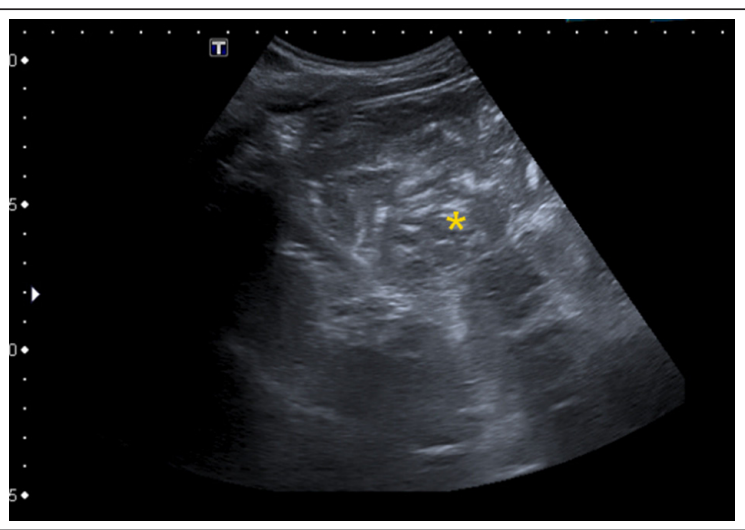

Figure 1. A 41 year-old man with long-term intermittent abdominal pain, bloating and vomiting. Ultrasound study shows abnormal gastric dilatation with food retention $\left({ }^{*}\right)$ even though in fasting, preventing a proper assessment of retroperitoneal structures.

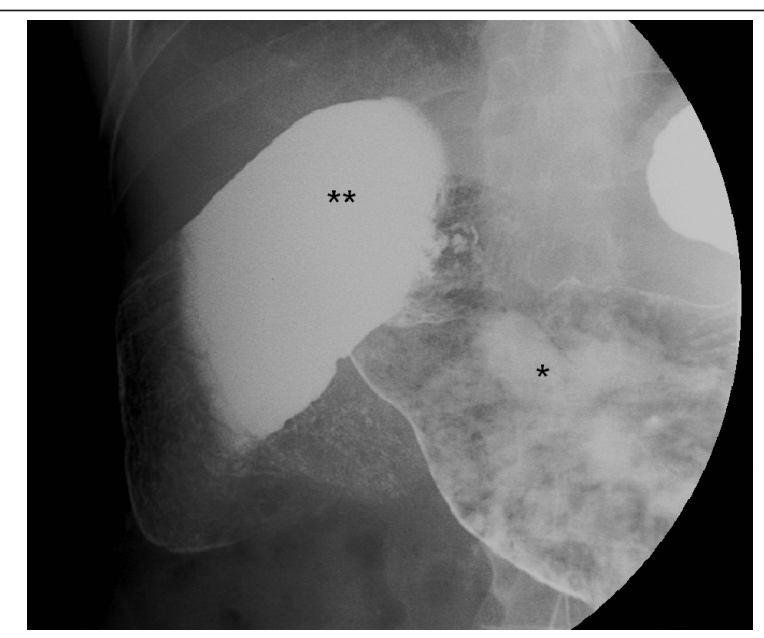

Figure 2. Barium gastrointestinal transit showed already known gastric dilatation with food retention $\left({ }^{*}\right)$ extended to the proximal duodenum without bulbar deformity $\left({ }^{* *}\right)$.

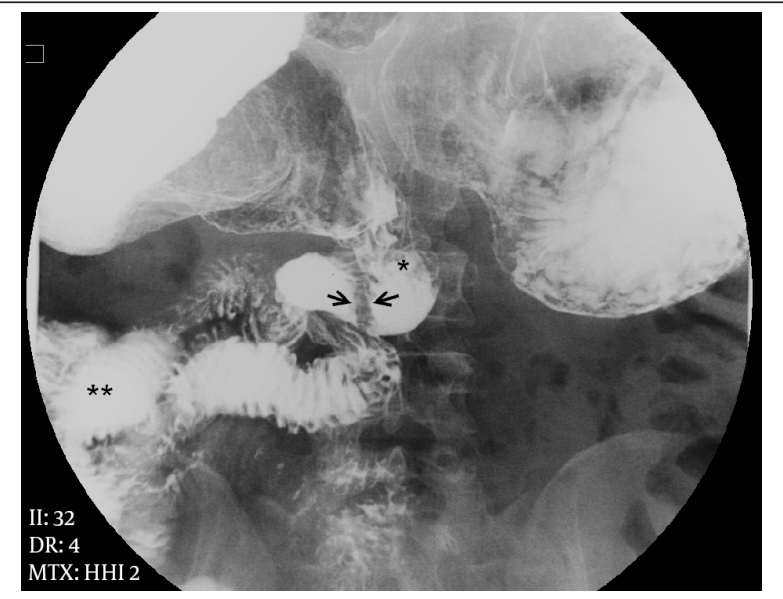

Figure 3. Barium gastrointestinal transit showed a depressed duodenojejunal flexure with variation of midgut usual trajectory, being the fourth part of duodenum downward instead of upward $\left({ }^{*}\right)$, locating jejunal loops in the right upper quadrant $\left(^{* *}\right)$. At duodenojejunal transition, a linear mark (arrows) is seen corresponding to the Ladd's band; note that from this point, loop caliber becomes normal.
Abdominopelvic CT study was performed with a multidetector row scanner (BrightSpeed 16; GE Medical Systems, Milwaukee, USA). Negative oral contrast (water) was administered. Angiographic images were obtained after intravenous injection of iodinated contrast, at injection rate of $3 \mathrm{~mL} / \mathrm{s}$, using bolus test tracking located at descending aorta. Multiplanar and volume rendering reconstructions were used for interpretation. These showed gastric and small intestine dilatation with food retention, abnormal rotation of the small intestine and vessels around the root of the mesentery (whirl sign), beak-like appearance and cecum located in the right upper quadrant, confirming the diagnosis of midgut malrotation and volvulus. Collateral intestinal perfusions supplied by an inferior mesenteric artery via a hypertrophic Riolan arc and by the jejunal branches of the celiac artery were also seen. An enlarged superior mesenteric vein (SMV) in abnormal location posterior and left of the superior mesenteric artery (SMA) was found (Figure 4). Angiographic images depicted clockwise twisting of SMA, with corkscrew appearance, showing the "barber pole sign" in the volume rendering three-dimensional reconstructions (Figure 5).

With the diagnosis of intermittent partial bowel obstruction due to midgut malrotation with volvulus, the patient was admitted for scheduled surgical treatment, including duodenal release with supraantral duodenal and gastric partitioning performing omega-foot bypass loop, colonpexia and appendectomy. The patient had an uneventful postoperative recovery and was discharged after one week of postsurgical stay without any incidence. He remained asymptomatic and had significant improvement in his quality of life.

\section{Discussion}

Midgut malrotation is a rare diagnosis, with a reported incidence lower than $0.2 \%$, and is usually asymptomatic. When symptoms occur, normally present during the first year after birth, although a few of them may remain quiescent during childhood and appear in adult life, presenting with long-term unspecific abdominal complains such as pain, bloating and vomiting due to intermittent volvulation, a dynamic lesion with frequent spontaneous remissions and relapses. Less frequently, patients may present with acute bowel obstruction. Both its low incidence and unspecific symptoms make this pathologic entity rarely considered on clinical diagnosis. This explains often diagnostic delay, accounting for many years of abdominal complains before diagnosis (4). Midgut malrotation is an embryological anomaly. From the fifth to the tenth week of fetal development, the embryonic gut undergoes rapid elongation exceeding the capacity of the abdominal cavity causing umbilical herniation. During this period, midgut goes through counter-clockwise rotation in a 270 degree, gradually returning to the abdominal cavity. Failure in this process can cause different degrees of anomalies in the location of small bowel, 

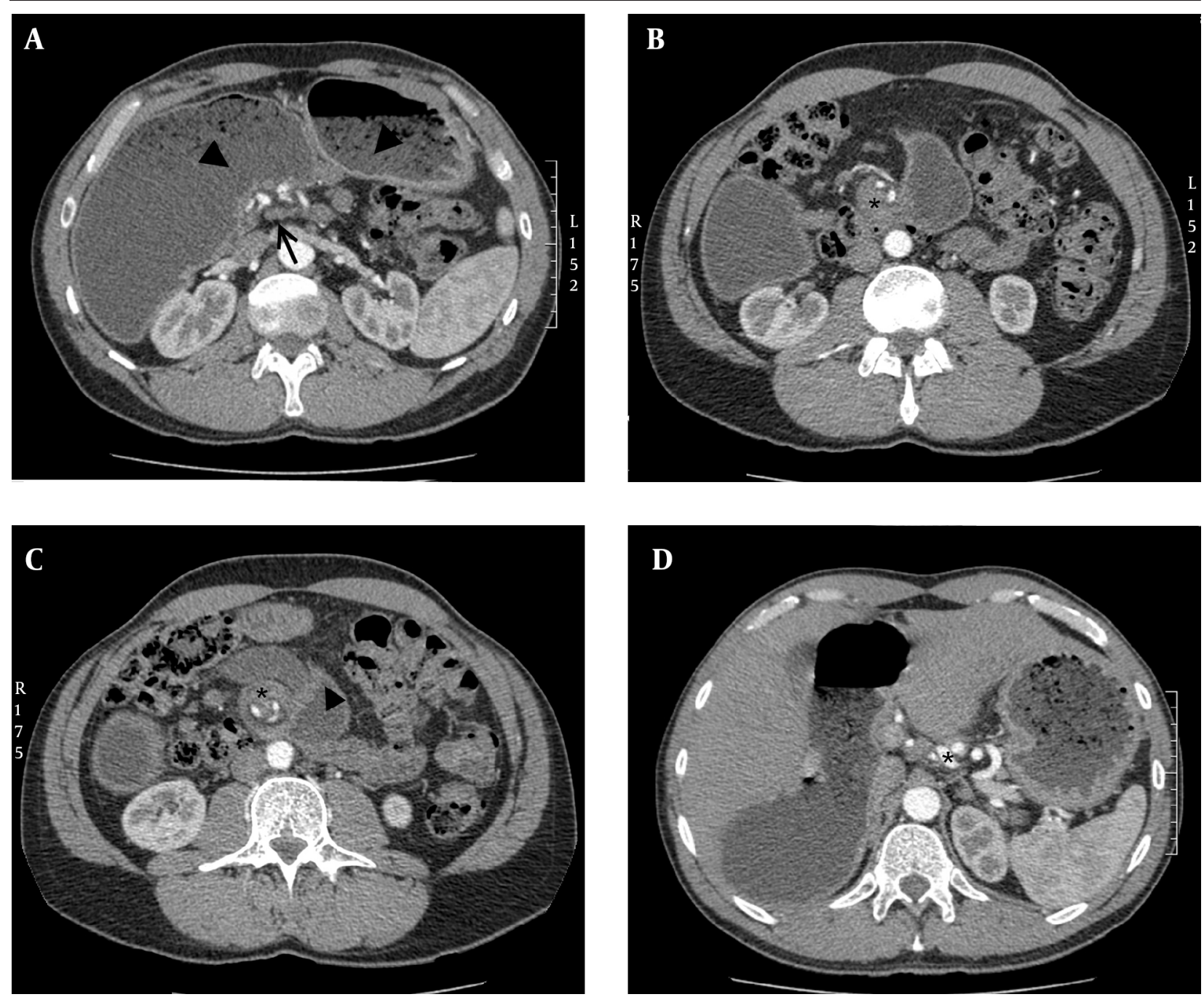

Figure 4. Abdominal CT shows proximal superior mesenteric vein posterior and left of the superior mesenteric artery (arrow, A) gastric dilatation with food retention (arrowhead, A), abnormal rotation of the small intestine around the root of the mesentery $\left({ }^{*}, \mathrm{~B}\right.$ and $\left.\mathrm{C}\right)$, and collateral intestinal perfusion supplied by hypertrophic jejunal branches of celiac trunk $\left({ }^{*}, \mathrm{D}\right)$.
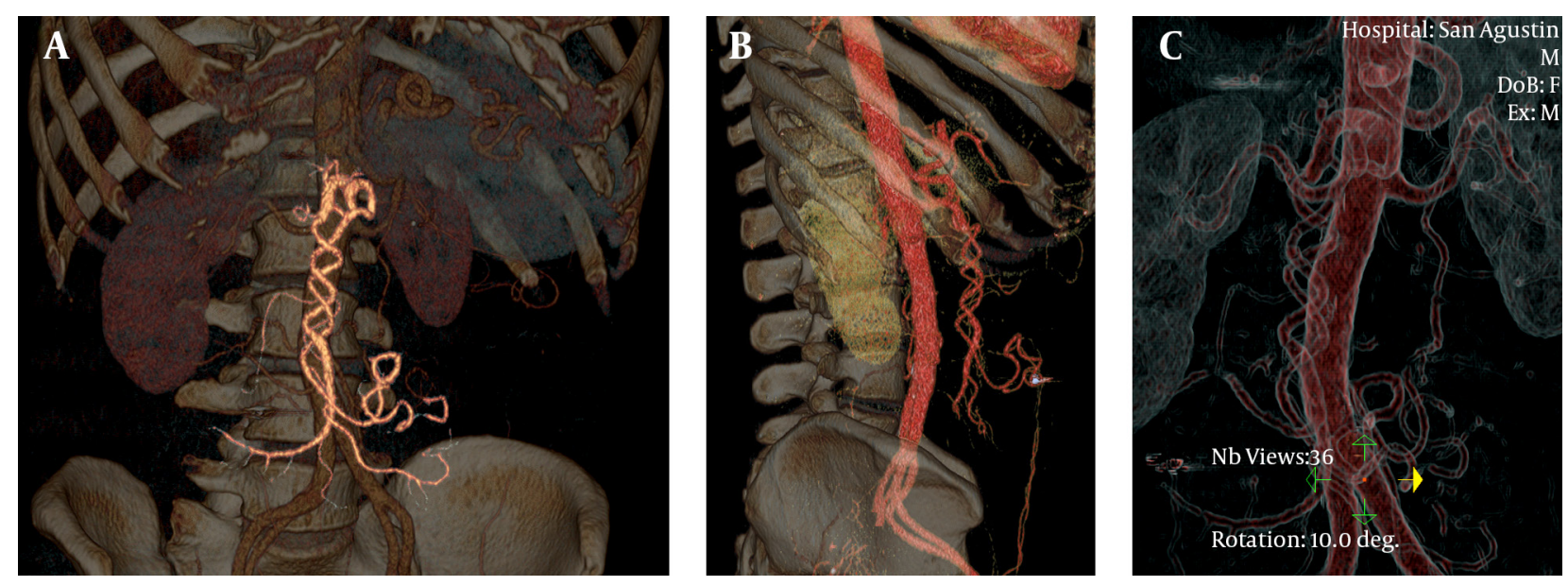

Figure 5. Angiographic CT images, performing volume rendering 3D reconstructions (A, B and C), depicts clockwise twisting of both superior mesenteric artery and gastroduodenal branch of the celiac trunk, corkscrew appearance, showing the barber pole sign. 
caecum, colon and appendix. Peritoneal fibrous bands fixing the duodenum, known as the Ladd's bands, may persist and eventually compress the duodenum causing extrinsic obstruction. Malrotation results in malposition of the bowel and malfixation of the mesentery, resulting in a narrow pedicle at the mesenteric attachment, which facilitates volvulation.

Imaging techniques play an important role in the diagnosis of this pathologic condition. The actual widespread increase of diagnostic imaging studies may increase asymptomatic midgut malrotation diagnosis. In symptomatic patients, imaging techniques may reveal a nonsuspected diagnosis, shortening the time gap between debut of symptoms and surgical treatment if required.

Diagnosis hints of midgut malrotation may be recognized by different imaging techniques. Plain abdominal radiography is often the first imaging study performed in these patients, but conventional radiography has low utility. Nevertheless, there are a few findings such as abnormal gas-bowel distribution, right sided jejunal loops and absent colon in the right lower quadrant, which may raise the suspicion of abdominal anomaly and lead to more specific imaging studies.

Upper gastrointestinal barium contrast study is highly sensitive for detection of midgut malrotation. It usually shows duodenum and duodenojejunal flexure in the right half of the abdomen, as well as abnormal location of jejunum loops. However, these studies have lower sensitivity for detecting associated complications and are not very specific; a normal barium study cannot rule out gut malrotation (5).

Ultrasound study, especially indicated in pediatric patients, may reveal abnormal location and caliber of bowel loops; in some cases a dilated duodenum with distal tapering is appreciable. Using color Doppler atypical situation of SMA may be shown as well as twisted mesentery around the SMA; these features are known as the "whirlpool" sign, however in adult patients, technical difficulties limit its use (6).

Nowadays, CT has been considered the most useful imaging technique for demonstrating adult midgut malrotation with or without volvulus; it can depict SMV in anomalous location posterior and left of the SMA (7); this is a frequent finding but is not entirely diagnostic. Patients with malrotation may have a normal SMA-SMV orientation, and patients with abnormal SMA-SMV relationship may not have malrotation corresponding to normal variant (8). In addition, abnormal disposition of midgut and duodenum not crossing the spine can be shown. In case of malrotation with volvulus, besides the above findings, CT can show small bowel and mesentery twisted around the narrow SMA pedicle showing the "whirl-pool sign", first described by Fisher (9). Also a range of abnormalities like dilatation of small intestine, circumferential bowel thickening, halo appearance, beak-like appearance or closed loops can be seen (10). In specific circumstances, abdominal MRI may be performed (11) revealing similar anatomical findings.
Mesenteric angiography has nowadays limited use in diagnostic management of suspected malrotation, because of invasive nature and high cost, but in the past, it was used to demonstrate abnormal mesenteric vessels, with typical corkscrew appearance of whirling SMA and its branches, showing the "barber pole sign" (3). In addition to this finding, tapering or abrupt termination of mesenteric vessels, prolonged contrast transit time, absent venous opacification, or dilated SMV can be detected. Angiographic CT volume rendering reconstructions can depict similar findings helping in surgical approach (12), although in articles previously cited, similar cases were reported, value of volume rendering reconstructions replacing diagnostic angiography is the outstanding point in this case report.

When symptomatic, midgut malrotation requires surgical intervention whether open or laparoscopic, habitually following the Ladd's Procedure or any of its variations. Meanwhile, management of asymptomatic patients is controversial (13); there are studies that reveal low risk of volvulus at follow-up (14) as well as studies that recommend surgery to prevent catastrophic complications (15). Opinion of patient, clinical condition and potential risks of the procedure weighted against benefits should be considered in each case.

\section{Authors' Contributions}

All authors fully participated and accepted responsibility for this work.

\section{References}

1. Dietz DW, Walsh RM, Grundfest-Broniatowski S, Lavery IC, Fazio VW, Vogt DP. Intestinal malrotation: a rare but important cause of bowel obstruction in adults. Dis Colon Rectum. 2002;45(10):1381-6.

2. de Korte N, Grutters CT, Snellen JP. Small bowel volvulus diagnosed by the CT whirl sign.J Gastrointest Surg. 2008;12(8):1469-70.

3. Buranasiri SI, Baum S, Nusbaum M, Tumen $H$. The angiographic diagnosis of midgut malrotation with volvulus in adults. Radiol ogy. 1973;109(3):555-6.

4. Fu T, Tong WD, He YJ, Wen YY, Luo DL, Liu BH. Surgical management of intestinal malrotation in adults. World J Surg. 2007;31(9):1797-803.

5. Pickhardt PJ, Bhalla S. Intestinal Malrotation in Adolescents and Adults: Spectrum of Clinical and Imaging Features. Am J Roentgenol. 2002;179(6):1429-35.

6. Fukumoto A, Tanaka S, Imagawa H, Shishido T, Oka S, Yoshida S, et al. Usefulness and limitations of transabdominal ultrasonography for detecting small-bowel tumors. Scand J Gastroenterol. 2009;44(3):332-8.

7. Nichols DM, Li DK. Superior mesenteric vein rotation: a CT sign of midgut malrotation. AJR Am J Roentgenol. 1983;141(4):707-8.

8. Zissin R, Rathaus V, Oscadchy A, Kots E, Gayer G, Shapiro-Feinberg $M$. Intestinal malrotation as an incidental finding on CT in adults. Abdom Imaging. 1999;24(6):550-5.

9. Fisher JK. Computed tomographic diagnosis of volvulus in intestinal malrotation. Radiology. 1981;140(1):145-6.

10. Feng ST, Chan T, Sun CH, Li ZP, Guo HY, Yang GQ, et al. Multiphasic MDCT in small bowel volvulus. EurJ Radiol. 2010;76(2):e13-8.

11. Kouki S, Fares A, Alard S. MRI whirpool sign in midgut volvulus with malrotation in pregnancy. JBR-BTR. 2013;96(6):360-1. 


\section{Garcelan-Trigo JA et al.}

12. Bozlar U, Ugurel MS, Ustunsoz B, Coskun U. CT angiographic demonstration of a mesenteric vessel whirlpool in intestinal malrotation and midgut volvulus: a case report. Korean J Radiol. 2008;9(5):466-9.

13. Emanuwa OF, Ayantunde AA, Davies TW. Midgut malrotation first presenting as acute bowel obstruction in adulthood: a case report and literature review. World JEmerg Surg. 2011;6(1):22.
14. Choi M, Borenstein SH, Hornberger L, Langer JC. Heterotaxia syndrome: the role of screening for intestinal rotation abnormalities. Arch Dis Child. 2005;90(8):813-5.

15. Matzke GM, Dozois EJ, Larson DW, Moir CR. Surgical management of intestinal malrotation in adults: comparative results for open and laparoscopic Ladd procedures. Surg Endosc. 2005;19(10):1416-9. 